\section{Aboriginal curriculum}

\section{framework developed}

I n Calgary, Alberta, Dr. Linden Crowshoe challenges medical students to confront their stereotypes about the indigenous peoples of Canada through the use of interactive political theatre. At the Northern Ontario School of Medicine, first-year students are sent in teams to live in Aboriginal communities for a month to experience the culture first hand. And McMaster University draws on expertise at the nearby Six Nations reserve to help educate medical students.

These are some of the "best practices" that will likely be highlighted in a Web-based resource that the Association of Faculties of Medicine of Canada plans to create to accompany a new curriculum framework in First Nations, Inuit and Métis health.

Developed in collaboration with the Indigenous Physicians Association of Canada, the framework is designed to educate students about the continuing impact of colonization - "with its resulting racism, discrimination and marginalization" - on the health of indigenous peoples. The goal is more competent and more equitable medical care because Aboriginal patients "are often faced with physicians who might not recognize, acknowledge and address some of the barriers they face to improving their health."

The framework also aims to help create a more "culturally safe" environment at the country's 17 medical schools. Dr. Marcia Anderson, who graduated in 2002 and is now medical officer of health for Northern Manitoba, recalls encountering some "frankly racist" attitudes during her training. A senior obstetrical resident, for example, offered the view that native peoples should simply stop reproducing, she said.

Discrimination in the provision of medical care for Aboriginal peoples in Canada has been well documented. For example, they receive kidney transplants at about half the rate of the general population, regardless of distance from transplant centres (Kidney Int 2006;70:924-30), even though high rates of diabetes in the population, and subsequent kidney failure rates, are far above the population average.

The new curriculum framework comes 3 years after the deans of Canada's medical schools agreed that the initiative, funded in part by Health Canada's First Nations Inuit Health branch, was needed to help educate students and medical faculty. It stresses that Aboriginal peoples are not to be considered one of many cultural groups in Canada, "but rather a distinct constitutionally recognized peoples with Aboriginal and treaty rights."

In her first year at the Northern Ontario School of Medicine, Anna Soverio spent a month in Eabametoong (Fort Hope), a fly-in reserve about $300 \mathrm{~km}$ north of Thunder Bay, Ontario, with a population of about Iooo and a nursing station. Soverio, who is now entering third year and plans to stay and work in the north, says the experience taught her to think carefully and be creative about treatment plans she will propose.

"Good nutrition is important given high rates of diabetes, so you might recommend eating lots of fruits and vegetables, but on a good day, there are limited reserves at the stores and the cost is very high. And if someone, for example, requires rehab, there are no physiotherapists."

But it's not just doctors who practice in more remote areas who need to be aware of circumstances and cultural issues - Anderson notes that about half of Canada's Aboriginal population now live in the urban areas.

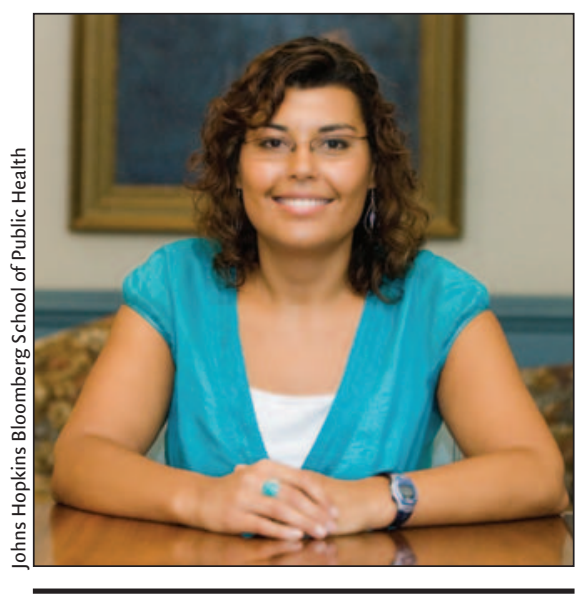

Dr. Marcia Anderson, medical officer of health for Northern Manitoba, encountered "racist" attitudes as a student.
There are no official counts of the number of Aboriginal doctors or medical students in Canada, and it is up to students to self-identify, Anderson says. She estimates there are about 150 practising physicians and that students comprise about $\mathrm{I} \%$ of those now enrolled in medical school, while First Nations, Inuit and Métis account for $4 \%$ of the Canadian population.

Some medical schools have recently placed a priority on attracting Aboriginal students. For the past few years McMaster, for example, has admitted II or 12 such students into each first-year class of 150 , compared to "one every few years" in the past, says undergraduate dean Dr. Alan Neville.

Neville notes the First Nations, Inuit and Métis Health curriculum framework is a "guiding document" and the fifth in a series of such collaboratively developed frameworks; the first 4 covered public health, end of life care, gender health and complementary medicine.

The newest framework, though, differs in that it necessitates significant relationship building with local Aboriginal communities, says Neville, cochair of the Aboriginal health curriculum subcommittee. "We need expertise to teach it and that means working with communities to get people to feel comfortable teaching at university." Another challenge is that "this will not be all classroom stuff. It also has to be experiential."

Many schools have electives in Aboriginal health, but the ultimate goal is to incorporate this learning into the core curriculum, not make it a stand alone unit, Neville says. The framework, which was developed using the CanMEDs model of 7 core competencies, recommends that physicians be able to describe the impact of, for example, residential schools on intergenerational health. The "sad reality" is that in most cases medical students will have to be taught basics about the history of indigenous peoples, since most have not learned about this in their previous educational experiences, notes Barbie Shore, a project manager for the Association of Faculties of Medicine of Canada. - Ann Silversides, CMAJ

DOI:Io.I503/cmaj.080803 